\title{
UNIVERSAL HIDDEN SUPERSYMMETRY IN CLASSICAL MECHANICS AND ITS LOCAL EXTENSION凸
}

\author{
E. Gozzi \\ Dept. of Theor. Physics, University of Trieste \\ Strada Costiera 11, Miramare-Grignano \\ 34014 Trieste, Italy \\ INFN Sezione di Trieste \\ gozzi@trieste.infn.it
}

\begin{abstract}
We review here a path-integral approach to classical mechanics and explore the geometrical meaning of this construction. In particular we bring to light a universal hidden BRS invariance and its geometrical relevance for the Cartan calculus on symplectic manifolds. Together with this BRS invariance we also show the presence of a universal hidden genuine non-relativistic supersymmetry. In an attempt to understand its geometry we make this susy local following the analogous construction done for the supersymmetric quantum mechanics of Witten.
\end{abstract}

\section{Introduction}

The discovery of supersymmetry [1], first at the pure algebraic level then in two dimensions and finally in four dimensions both in its non-linear and linear versions, has been one of the most fascinating discovery of the last 30 years. Besides softening the ultraviolet behaviour of theories of fermions coupled with bosons, supersymmetry offered a way out, in its unbroken phase, to the cosmological constant problem.

Unfortunately the spectrum of particles known to-day indicates that supersymmetry must be broken. This brings back the cosmological constant problem unless the breaking of this special symmetry manages to avoid the usual theorems on symmetry breaking. In order to explore that possibility it is crucial to understand supersymmetry at a deeper level.

Supersymmetry, soon after its discovery, was made local [2] producing theories of gravity that raised the hopes of not being afflicted by the problem of the non-removable infinities which plagued Einstein gravity. Despite the huge amount of work done on supergravity and later on superstrings, still the goal of a finite or renormalizable theory of gravity seems not to be near.

\footnotetext{
${ }^{1}$ This work is dedicated to the memory of Dmitrij Vasil'evich Volkov and to all those who are struggling to have their work recognized. I wish to warmly thank the organizers of the Volkov Memorial for the heroic effort of organizing it during a freezing winter in Ukraine.
} 
All of the above mentioned issues seem to indicate that supersymmetry is very important but its roots should be understood better. It is our opinion that these roots are deeply geometrical.

In this respect there has been a 1-D model, proposed by Witten in 1981 [3], which has been a toy model for exploring several issues connected both with supersymmetry breaking and with geometry [4]. The model has the limit of being just a model reproducing no aspect whatsoever of the natural world, except some phenomenological stochastic dynamics [5]. Quite independently of that model, the present author, together with M.Reuter and W.D.Thacker, has tried [6] to give a path-integral representation to Classical Mechanics (CM) and the outcome has been a superlagrangian which has both a physical and geometrical meaning and it is not just a toy model. So we feel that some geometrical issues regarding supersymmetry could be tackled better using our Lagrangian. In fact it turns out that the 1-D superhamiltonian produced by this path-integral is nothing else than the Lie-derivative of the Hamiltonian flow and it has both a universal BRS-AntiBRS-like invariances and also a universal genuine non-relativistic supersymmetry [7]. The BRS-AntiBRS invariances have been interpreted geometrically as being the exterior derivative and co-derivative on symplectic spaces, and all the standard Cartan calculus can be reproduced via our formalism. As we said before there is also a genuine supersymmetry whose physical meaning has been studied in [7] as being related to the ergodicity-non-ergodicity of the Hamiltonian system under consideration. That supersymmetry [f] had also other nice features. For example, due to the non-positivity of the Hilbert space, it could be broken without lifting the vacuum from zero and this can be very important for the cosmological constant problem. Being this susy universal it could be extracted not only from a point particle dynamics but also from any classical field theory once they are formulated via a Lie-brackets kind of formalism. Of course it would not be a relativistic supersymmetry even for relativistic field theories because it gives a privileged role to the forth component of space-time. It might be possible to made it relativistic if one uses the De Donder-Weyl \& formulation of field-theory, but this has still to be explored in details [6]. Of course we would obtain in that case a susy at the Lie-bracket level in which each boson and fermion present in the basic theory, whose Hamiltonian will act as a superpotential for our superhamiltonian, will get a partner and produce in that manner a huge pletora of fields which will live in a sort of shadow world degenerate with our world. The meaning of these partners would be purely geometrical as it is clear from 66. It would be interesting if these shadow world, made only of auxiliary geometrical fields, had to be included in the counting needed for the cosmological constant 9.

To better understand the geometry behind this supersymmetry, in this brief note we take a first step in that direction by gauging it in the simple case of the point particle dynamics. 


\section{Path-Integral for Classical Mechanics}

This section is meant to be a very quick and incomplete review of the pathintegral approach to CM. For more details the reader should study [6]. The "propagator" which gives the classical probability for a particle to be at the point $\phi_{2}$ at time $t_{2}$, if it it was at the point $\phi_{1}$ at time $t_{1}$, is just a delta function $P\left(\phi_{2}, t_{2} \mid \phi_{1}, t_{1}\right)=\delta^{2 n}\left(\phi_{2}-\Phi_{c l}\left(t_{2}, \phi_{1}\right)\right)$ where $\Phi_{c l}\left(t, \phi_{0}\right)$ is a solution of Hamilton's equation $\dot{\phi}^{a}(t)=\omega^{a b} \partial_{b} H(\phi(t))$ subject to the initial conditions $\phi^{a}\left(t_{1}\right)=\phi_{1}^{a}$. Here $H$ is the conventional Hamiltonian of a dynamical system defined on some phase-space $\mathcal{M}_{2 n}$ with local coordinates $\phi^{a}, a=1 \cdots 2 n$ and constant symplectic structure $\omega=\frac{1}{2} \omega_{a b} d \phi^{a} \wedge d \phi^{b}$. Slicing in infinitesimal parts the time interval $\left[t_{2}-t_{1}\right]$ above and doing standard manipulations [6], which for brevity we do not reproduce here, it is possible to give a path-integral representation to the transition probability above:

$$
P\left(\phi_{2}, t_{2} \mid \phi_{1}, t_{1}\right)=\int_{\phi_{1}}^{\phi_{2}} \mathcal{D} \phi \mathcal{D} \lambda \mathcal{D} c \mathcal{D} \bar{c} \exp i \int_{t_{1}}^{t_{2}} \widetilde{\mathcal{L}}
$$

where $\widetilde{\mathcal{L}} \equiv \lambda_{a}\left[\dot{\phi}^{a}-\omega^{a b} \partial_{b} H(\phi)\right]+i \bar{c}_{a}\left(\delta_{b}^{a} \partial_{t}-\partial_{b}\left[\omega^{a c} \partial_{c} H(\phi)\right]\right) c^{b}$. The $\lambda_{a}$ are c-number auxiliary variables while the $c^{a}, \bar{c}_{a}$ are Grassmannian auxiliary variables, so the new space is an enlargement of the phasespace from $2 n$ dimensions to $8 n$. The standard equations of motion for $\phi^{a}$ can be obtained from the variation with respect to $\lambda_{a}$ of the above Lagrangian. They can also be obtained by the introduction of the the associated Hamiltonian $\widetilde{\mathcal{L}}=\lambda_{a} \dot{\phi}^{a}+i \bar{c}_{a} \dot{c}^{b}-\widetilde{\mathcal{H}}$, with $\widetilde{\mathcal{H}}$ given by $\widetilde{\mathcal{H}}=\lambda_{a} h^{a}+i \bar{c}_{a} \partial_{b} h^{a} c^{b}$ where $h^{a}(\phi) \equiv \omega^{a b} \partial_{b} H(\phi)$ are the components of what is called the the Hamiltonian vector field [10]. To get the eqs. of motion via $\widetilde{\mathcal{H}}$ one needs also an extended Poisson bracket structure $(e p b)$ in the above mentioned enlarged space. It is easy to see that they are given by $\left\{\phi^{a}, \lambda_{b}\right\}_{e p b}=\delta_{b}^{a},\left\{c^{a}, \bar{c}_{b}\right\}_{e p b}=-i \delta_{b}^{a}$, all others $=0$ Note that these are different from the normal Poisson brackets on $\mathcal{M}$ which were $\left\{\phi^{a}, \phi^{b}\right\}_{p b}=\omega^{a b}$. Via the extended Poisson brackets above we obtain from $\widetilde{\mathcal{H}}$ the same equations of motion as those which one would obtain (at least for $\phi^{a}$ ) from $H$ via the normal Poisson brackets: $\left\{\phi^{a}, H\right\}_{p b}=\left\{\phi^{a}, \widetilde{\mathcal{H}}\right\}_{e p b}$. Regarding the $c^{a}$ one can easily see [6] that they can be identified with the forms $c^{a}=d \phi^{a}$, so the coordinates $\left(\phi^{a}, c^{a}\right)$ can be thought as labelling the cotangent bundle $T^{*} \mathcal{M}$ to phase-space. It is also easy 6 , via the operatorial formulation of CM, to realize that the $\lambda_{a}, \bar{c}_{a}$ fields are instead a basis of the tangent bundle to the previous space. So the $8 n$ variables $\left(\phi^{a}, c^{a}, \lambda_{a} \cdot \bar{c}_{a}\right)$ are a set of coordinates for the tangent bundle to the cotangent bundle to phase-space $T\left(T^{*} \mathcal{M}\right)$. This gives a complete geometrical description of all the various auxiliary variables which we had to introduce and makes this not just a model but something more fundamental. It is also possible [6] to make a correspondence between higher forms and polynomials in $c^{a}$ and between antisymmetric multivector fields 10 and polynomials in $\bar{c}_{a}$. We will indicate this correspondence via a $\widehat{(\cdot)}$ sym- 
bol: $F^{(p)}=\frac{1}{p !} F_{a_{1} \cdot a_{p}} d \phi^{a_{1}} \wedge \cdots \wedge d \phi^{a_{p}} \Longrightarrow \widehat{F}^{(p)}=\frac{1}{p !} F_{a_{1} \cdots a_{p}} c^{a_{1}} \cdots \wedge c^{a_{p}}$, $v^{(p)}=\frac{1}{p !} V^{a_{1} \cdots a_{p}} \partial_{a_{1}} \wedge \cdots \wedge \partial_{a_{p}} \Longrightarrow \widehat{V}^{(p)}=\frac{1}{p !} \bar{c}_{a_{1}} \cdots \bar{c}_{a_{p}}$. Before proceeding further we should also point out that the $\widetilde{\mathcal{H}}$ presents some universal invariance whose charges are the following [6]: $Q \equiv i c^{a} \lambda_{a}$, $\bar{Q} \equiv i \bar{c}_{a} \omega^{a b} \lambda_{b}, \quad Q_{g} \equiv c^{a} \bar{c}_{a}, \quad K \equiv \frac{1}{2} \omega_{a b} c^{a} c^{b}, \quad \bar{K} \equiv \frac{1}{2} \omega^{a b} \bar{c}_{a} \bar{c}_{b} . \quad$ Using the correspondence indicated above it is then possible to rewrite all the normal operations of the Cartan calculus 10, like doing an exterior derivative on forms $d F$, or doing an interior product between a vector field and a form $i_{v} F$, or building the Lie-derivative of a vector field $l_{h}$, by just using polynomials in $c$ and $\bar{c}$ together with the extended Poisson brackets structure and the charges built above. These rules, which we called $\{\cdot, \cdot\}_{e p b}$-rules, are summarized here: $d F^{(p)} \Longrightarrow i\{Q, \widehat{F}\}_{e p b}, \quad i_{v} F^{(p)} \Longrightarrow$ $i\left\{\widehat{v}, \widehat{F}^{(p)}\right\}_{e p b} ; \quad l_{h} F=d i_{h} F+i_{h} d F \Longrightarrow-\{\widetilde{\mathcal{H}}, \widehat{F}\}_{e p b}, \quad p F^{(p)} \Longrightarrow$ $i\left\{Q_{g}, \widehat{F}\right\}_{e p b}, \omega(v, \cdot) \equiv v^{b} \Longrightarrow i\{\bar{K}, \widehat{V}\}_{e p b}, \quad(d f)^{\sharp} \Longrightarrow i\{\bar{Q}, f\}_{e p b}$ where the last three operations indicated above are, respectively, multiplying a form $F^{(p)}$ by its degree $p$, mapping a vector field $V$ into its associated one form $V^{b}$ via the symplectic form, and building the associated Hamiltonian vector field $(d f)^{\sharp}$ out of a function $f$. One sees from above that the various abstract derivations of the Cartan calculus are all implemented by some charges acting via the epb-brackets. From the third relation above one also can notice that the Lie-derivative of the Hamiltonian vector field of time evolution becomes nothing else than our $\widetilde{\mathcal{H}}$, thus confirming that the weight-function of our classical path-integral, generated by just a simple Dirac delta, is the right geometrical object associated to the time-evolution.

\section{Universal Supersymmetry}

The universal charges indicated in the previous section, $Q, \bar{Q}, Q_{g}, K, \bar{K}$, have all a geometrical meaning [6] as it is clear from the previous section. In particular $Q$ plays the role of the exterior derivative while $\bar{Q}$ is the corresponding operation on vector fields. The reason they are conserved is because the exterior derivative anticommutes with the Lie-derivative. If we interpret $\widetilde{\mathcal{L}}$ as a 1-D field theory, then $Q, \bar{Q}$ are also analog of BRSantiBRS charges 11] because their graded epb-brackets are: $\{Q, \bar{Q}\}_{e p b}=$ $0,\{Q, Q\}_{e p b}=0,\{\bar{Q}, \bar{Q}\}_{e p b}=0$. Besides these BRS-charges, there are also other charges conserved under $\widetilde{\mathcal{H}}$ which make up, once combined with the $Q$ and $\bar{Q}$, a genuine supersymmetry. They are []] $N \equiv c^{a} \partial_{a} H$, $\bar{N} \equiv \bar{c}_{a} \omega^{a b} \partial_{b} H$. The brackets among themselves and with $Q$ and $\bar{Q}$ are all zero except the following ones $\{Q, \bar{N}\}_{e p b}=\widetilde{\mathcal{H}} \quad, \quad\{i \bar{Q}, i N\}_{e p b}=\widetilde{\mathcal{H}}$. This is a $\mathrm{N}=4$ supersymmetry. In fact these charges can be combined in the following four independent ones: $Q_{(1)} \equiv \frac{i(Q-\bar{N})}{\sqrt{2}}, Q_{(2)} \equiv \frac{(Q+\bar{N})}{\sqrt{2}}$, $Q_{(3)} \equiv \frac{i(\bar{Q}+N)}{\sqrt{2}}, Q_{(4)} \equiv \frac{(\bar{Q}-N)}{\sqrt{2}}$, all of which give $\left\{Q_{(i)}, Q_{(i)}\right\}_{e p b}=\widetilde{\mathcal{H}}$ with $(i)=(1) \cdots(4)$. This universal supersymmetry of CM has a nice physical interpretation deeply related to the fact that we are describing a 
classical system. It basically says [7] that the spontaneous breaking of the invariance generated by the linear combinations of some of the $Q_{(i)}$ above occurs for non-ergodic systems while ergodic systems have that susy (at constant energy) always unbroken.

We would like here to explore instead the geometrical meaning of the susy charges. The reason is that all charges we found previously had a nice geometrical interpretation, so also the $Q_{(i)}$ must have one. To better understand that, a first question we asked ourselves is why God, in creating such a fundamental object as the Lie-derivative $\widetilde{\mathcal{H}}$, did not think of making the Susy local. To a mathematician this way of studying the geometrical meaning of a charge may sound strange, but not to a physicist. So the first thing we decided to do is to go from the Lie-derivative $\widetilde{\mathcal{H}}$ (or its lagrangian $\widetilde{\mathcal{L}}$ ), to a new one $\widetilde{\mathcal{H}}_{g}$ (or $\widetilde{\mathcal{L}}_{g}$ ) which has that susy local. First (using the standard Noether technique 20 ) we do four local variations of $\widetilde{\mathcal{L}}$ generated by each of the four charges, $Q, \bar{Q}, N, \bar{N}$, which make up the four supersymmetric charges $Q_{(i)}$ and test which one of these variations just produces the associated charge itself multiplied by the time derivative of the symmetry parameter. The result [9] is that only the variation under $Q$ and $\bar{N}$ do this. So these are the only two symmetry generators which can be made local in the sense that, by introducing auxiliary gauge-fields in $\widetilde{\mathcal{L}}$, the local variation of $\widetilde{\mathcal{L}}$ can be absorbed by the variation of the gauge-fields. As only two of the four charges can be made local, we can say that from global $\mathrm{N}=4$ we go down to a local $\mathrm{N}=2$ susy. In term of the susy charges $Q_{(i)}$ those which can be made local are only $Q_{(1)}$ and $Q_{(2)}$. The next step is to obtain the expression of the local lagrangian $\widetilde{\mathcal{L}}_{g}$. It is easy 9] to find that: calling the "gauge" fields $e, \psi_{1}, \psi_{2}$ where $e$ is a c-number field while $\psi_{1}, \psi_{2}$ are Grassmannian ones, the $\widetilde{\mathcal{L}}_{g}$ is $\widetilde{\mathcal{L}}_{g} \equiv \lambda_{a} \dot{\phi}^{a}+i \bar{c}_{a} \dot{c}^{a}-e \widetilde{\mathcal{H}}+\psi_{1} \bar{N}+\psi_{2} Q$. It is not the first time that a susy or a BRS has been made local in a 1-D system 12] [13 14 but never for this action. The closest model for which it has been made local [13] is the supersymmetric quantum mechanics of Witten [3] (susyqm). The question now to ask is if $\widetilde{\mathcal{L}}_{g}$ has any geometrical meaning at all. To do that we have to explore the last three pieces present in $\widetilde{\mathcal{L}}_{g}$ which were not present in $\widetilde{\mathcal{L}}$. The author of ref. 13 indicates that there is a gauge transformation which would turn the field $e$ into the constant 1 while turning to zero both $\psi_{1}$ and $\psi_{2}$. In that manner the extra piece would disappear and $\widetilde{\mathcal{L}}_{g}$ would turn into $\widetilde{\mathcal{L}}$, at least for the susyqm. This fact does not happen for sure in our $\widetilde{\mathcal{L}}_{g}$ as we will show now and we have strong doubts it happens even in susyqm 13. The transformations of the gauge fields $e, \psi_{1}, \psi_{2}$ under the two local transformations associated to $\bar{N}$ and $Q$ (with parameters $\epsilon_{1}$, and $\left.\epsilon_{2}\right)$ are respectively $\left\{\delta_{1} \psi_{1}=\dot{\epsilon}_{1}, \delta_{1} \psi_{2}=0, \delta_{1} e=\epsilon_{1} \psi_{2}\right\}$ and $\left\{\delta_{2} \psi_{2}=\dot{\epsilon}_{2}, \delta_{2} \psi_{1}=0, \delta_{2} e=-\epsilon_{2} \psi_{1}\right\}$. As we have to bring, following 13], $\psi_{1}$ and $\psi_{2}$ to zero, the infinitesimal parameters which do that are $\epsilon_{1}(t)=-\int_{0}^{t} \psi_{1}\left(t^{\prime}\right) d t^{\prime}$ and $\epsilon_{2}(t)=-\int_{0}^{t} \psi_{1}\left(t^{\prime}\right) d t^{\prime}$. Using these parameters we have now at the same time to bring $e$ to zero. The combined transformation on $e$ would be: $\delta e=\left[\int_{0}^{t} \psi_{2} d t^{\prime}\right] \psi_{2}+\left[\int_{0}^{t} \psi_{1} d t^{\prime}\right] \psi_{1}$. This 
should be equal to $1-e$ to bring $e$ to zero but this is impossible because it would impose a relation between $e, \psi_{1}, \psi_{2}$ which does not exist. So this fact gives us the information that it is not possible to turn $\widetilde{\mathcal{L}}_{g}$ into $\widetilde{\mathcal{L}}$. The fields $e, \psi_{1}, \psi_{2}$ remain, they have no dynamic but they remain. They are the analog of the gravitons and gravitinos in 1-D. They are basically Lagrangian multipliers and the fact that they cannot be eliminated indicates that they have a role. In fact if we insert $\widetilde{\mathcal{L}}_{g}$ into $(1)$ we see that, once $e, \psi_{1}, \psi_{2}$ are integrated out, they impose some Dirac deltas constraints on the states between which we sandwich the propagator. This selection of states is related to the equivariant cohomology business [15] and this

should bring light to the geometrical meaning of $\widetilde{\mathcal{L}}_{g}$. More details will appear in 9].

\section{References}

[1] Yu.Gol'fand and E.Lichtman, JETP Lett 13 (1971) 323; J.-L.Gervais and B.Sakita, Nucl. Phys. B34 (1971)633; D.Volkov and V.Akulov, JETP Lett. 18 (1972) 438; J.Wess and B.Zumino, Nucl.Phys. B70 (1974) 39.

[2] D.Volkov and V.Soroka, Theor.Math.Phys.18 (1974) 28; D.Freedman, P.Van Nieuwenhuizen and S.Ferrara Phys.Rev D13 (1976) 3214; S.Deser and B.Zumino, Phys.Lett 62B (1976) 3214.

[3] E.Witten, Nucl.Phys. B188 513 (1981)

[4] E.Witten, Jour. Diff.Geom.17 (1982) 661;

[5] G.Parisi and N.Sourlas, Nucl.Phys. B206 (1982)321; S.Cecotti and L.Girardello, Ann.Phys.N.Y. 145 (1983) 81;

[6] E.Gozzi, MPI-PAE/Pth 47/86 Slac List PPF 8633; E.Gozzi, Phys. Lett.B 201 (1988) 525; E.Gozzi, M.Reuter, W.D.Thacker, Phys. Rev.D 40 (1989) 3363; ibidem D 46 (1992) 757; E.Gozzi and M.Reuter, Phys. Lett. 240B (1990) 137;

[7] E.Gozzi and M.Reuter, Phys.Lett.B 233 (1989) 383;

[8] H.Kastrup, Phys. Rep. 101 (1983) 1;

[9] E.Gozzi, work in progress;

[10] R.A.Abraham and J.Marsden, "Foundations of Mechanics" Benjamin, 1978, New York;

[11] C.Becchi, A.Rouet and R.Stora Ann.Phys. 98 (1976) 287;

[12] L.Brink et al.Phys.Lett.B 64 (1976) 435;

[13] E.Alvarez, Phys.Rev.D 29 (1984) 320;

[14] L.F.Urrutia and J.Zanelli, Jour.Math.Phys.31 (1990) 2271; C.Aragao de Carvalho, L.Baulieu, Phys.LettB 275, (1992) 323l; F.Bastianelli and L.Consoli, hep-th/9608065.

[15] S.Ouvry, R.Stora, P.van Baal, Phys.LettB 238 (1990) 291; 